\title{
Electroelastic moduli of piezoelectric polycrystals with bulk and film textures
}

\author{
Jiangyu Li, Martin L. Dunn
}

Jiangyu Li, Martin L. Dunn, "Electroelastic moduli of piezoelectric polycrystals with bulk and film textures," Proc. SPIE 4333, Smart Structures and Materials 2001: Active Materials: Behavior and Mechanics, (11 July 2001); doi:

10.1117/12.432743 


\title{
Electroelastic moduli of piezoelectric polycrystals with bulk and film textures
}

\author{
JiangYu Lia and Martin L. Dunn ${ }^{\mathrm{b}}$ \\ ${ }^{a}$ Division of Engineering and Applied Science, Caltech, Pasadena, CA 91125 \\ ${ }^{b}$ Department of Mechanical Engineering, University of Colorado, Boulder, CO 80309
}

\begin{abstract}
The effect of crystallographic texture on the electroelastic moduli of piezoelectric polycrystals has been studied using micromechanical modeling that makes use of the uniform field concept. An orientational averaging scheme has been developed for textured piezoelectric polycrystals, which, when combined with the conventional self-consistent approach, provides an estimate of the effective electroelastic moduli in terms of texture. In the special situation where the polycrystal exhibits a fiber texture, a class of uniform fields exist under certain crystal symmetries, so that the effective electroelastic moduli can be determined exactly. This is confirmed by the coincidence of the corresponding upper and lower bounds. Numerical results are presented for both cases and compared to known theoretical predictions where possible.
\end{abstract}

Keywords: Piezoelectric polycrystal, ceramic, thin film, texture

\section{INTRODUCTION}

Applications of piezoelectric ceramics and thin films have increased dramatically in recent years, fueled largely by their many uses in smart materials and structural systems. When a piezoelectric ceramic is fabricated by standard ceramic processing techniques, it is an aggregate of randomly oriented piezoelectric grains. No net macroscopic polarization is realized because the polarization directions are randomly oriented, and the material is not macroscopically piezoelectric. If the isotropic non-piezoelectric ceramic is subjected to a large electric field at high temperature (a process termed poling), the directions of polarization in many of the grains are permanently realigned resulting in a macroscopic spontaneous polarization and hence, piezoelectricity. The most prominent microstructural characteristic of thin films is the so-called columnar grain, i.e., highly oriented long needle-like grain. For materials in both bulk and film form, the grain orientation distribution is very important in determining the macroscopic behaviors of the polycrystalline aggregates. Furthermore, the same ideas apply at the subgrain level with regard to individual domains.

In this work, we study the effect of polycrystalline microstructure, especially texture, on the effective behavior of piezoelectric ceramics in both bulk and film forms ${ }^{1,2}$. An orientational averaging scheme is developed, which, when coupled with the self-consistent micromechanics approach, provides an estimate of the effective electroelastic moduli in terms of texture. In the special situation where the polycrystal exhibits a film texture, a class of uniform fields exist under certain crystal symmetries, so that the effective electroelastic moduli can be determined exactly. This problem is addressed by various authors from different aspects ${ }^{3-6}$. Although our scheme is developed strictly for linear constitutive behavior, when combined with an appropriate domain switch criteria, it can serve as the backbone for the analysis of more complex nonlinear behavior, as well as texture evolution under applied electrical or mechanical loading.

\section{MICROMECHANICS}

We consider the piezoelectric, and thus inherently anisotropic, analog of the uncoupled theory of elasticity, where the electric and elastic fields are fully coupled. The field variables and material moduli are represented either by conventional indicial notation or by bold characters. The constitutive equation for stationary linear response of a piezoelectric solid can be expressed as:

$$
\begin{aligned}
& \sigma_{p}=C_{p q} \varepsilon_{q}-e_{p k} E_{k} \\
& D_{i}=e_{i q} \varepsilon_{q}+\kappa_{i k} E_{k}
\end{aligned} .
$$


In eqs. (1) $\sigma_{p}$ and $\varepsilon_{p}$ are the stress and elastic strain, respectively; $D_{i}$ and $E_{i}$ are the electric displacement and field, respectively. $C_{p q}, e_{i q}$, and $\kappa_{i k}$ are the elastic stiffness tensor (measured in a constant electric field), the piezoelectric tensor, and the dielectric tensor (measured at a constant strain), respectively. The well known contracted notation for tensors is adopted ${ }^{7}$. We introduce the matrix representation for these quantities:

$$
\Sigma=\left[\begin{array}{l}
\sigma \\
\mathbf{D}
\end{array}\right], \quad \mathbf{Z}=\left[\begin{array}{c}
\varepsilon \\
-\mathbf{E}
\end{array}\right], \quad \mathbf{G}=\left[\begin{array}{cc}
\mathbf{C} & \mathbf{e}^{\mathrm{t}} \\
\mathbf{e} & -\kappa
\end{array}\right],
$$

the constitutive equations (1) can then be written as:

$$
\Sigma=\mathbf{G Z},
$$

where $\Sigma$ and $\mathbf{Z}$ are $9 \times 1$ column vectors representing the electroelastic field variables, $\mathbf{G}$ is $9 \times 9$ matrix representing the electroelastic moduli, and the superscript $t$ is used to denote the transpose of matrix. The electric field $-\mathbf{E}$ instead of $\mathbf{E}$ is used as independent variable because it allows the construction of symmetric moduli matrix, which proves to be advantageous.

Assuming statistical homogeneity for heterogeneous solid subjected to external loading consistent with the uniform fields $\mathbf{Z}^{0}$ on the boundary, the effective electroelastic moduli $\mathbf{G}^{*}$ can be defined as:

$$
<\Sigma>=\mathbf{G}^{*}<\mathbf{Z}>
$$

where $\left\langle\bullet>=\int(\bullet) d \Omega(\theta, \varphi, \phi)\right.$ denotes an orientational volume average, in which $(\theta, \varphi, \phi)$ are Euler angles ${ }^{8}$ describing the orientation of a grain $\mathrm{O}-\mathrm{X}_{1} \mathrm{X}_{2} \mathrm{X}_{3}$ in a global coordinate system $\mathrm{O}-\mathrm{x}_{1} \mathrm{X}_{2} \mathrm{X}_{3}$. Due to linearity we have:

$$
\mathbf{Z}(\theta, \varphi, \phi)=\mathbf{A}(\theta, \varphi, \phi) \mathbf{Z}^{0}
$$

where $\mathbf{A}(\theta, \varphi, \phi)$ is the concentration factor for grain at orientation $(\theta, \varphi, \phi)$, which is a function of microstructural parameters, such as grain shape, orientation, and the interactions among different grains. We can express the effective electroelastic moduli exactly as:

$$
\mathbf{G}^{*}=<\mathbf{G}(\theta, \varphi, \phi) \mathbf{A}(\theta, \varphi, \phi)>
$$

where $\mathbf{G}(\theta, \varphi, \phi)$ are the electroelastic moduli of a grain at orientation $(\theta, \varphi, \phi)$ expressed in the global coordinate system.

It is clear from eq. (6) that the estimation of effective electroelastic moduli depends on the estimation of the concentration factor $\mathbf{A}(\theta, \varphi, \phi)$. The simplest assumption for the concentration factor is $\mathbf{A}(\theta, \varphi, \phi)=\mathbf{I}$. This corresponds to assuming the existence of a uniform strain and electric field in the polycrystal subjected to an external loading, and gives:

$$
\mathbf{G}^{*}=<\mathbf{G}(\theta, \varphi, \phi)>
$$

Analogously, a uniform stress and electric displacement assumption gives:

$$
\mathbf{G}^{*}=<\mathbf{G}^{-1}(\theta, \varphi, \phi)>^{-1} .
$$

Equations (7) and (8) are the piezoelectric analog of the Voigt-Reuss averages in elastic solids ${ }^{9,10}$. The self-consistent approach ${ }^{11,12,1}$ assumes that the individual grains are embedded in an infinite matrix with yet to be determined effective electroelastic moduli $\mathbf{G}^{*}$, subjected to the yet to be determined external loading $\mathbf{Z}_{1}$ at the boundary. It follows that:

$$
\mathbf{G}^{*}=<\mathbf{G}(\theta, \varphi, \phi)\left(\mathbf{A}^{d i l}(\theta, \varphi, \phi)><\mathbf{A}^{d i l}(\theta, \varphi, \phi)>^{-1},\right.
$$

with 


$$
\mathbf{A}^{d i l}(\theta, \varphi, \phi)=\left\{\mathbf{I}+\mathbf{S}(\theta, \varphi, \phi) \mathbf{G}^{-1}(\theta, \varphi, \phi)\left[\mathbf{G}(\theta, \varphi, \phi)-\mathbf{G}^{*}\right]\right\}^{-1},
$$

where $\mathbf{S}(\theta, \varphi, \phi)$ is the piezoelectric Eshelby tensor ${ }^{13-15}$ for a grain at orientation $(\theta, \varphi, \phi)$ expressed in the global coordinate system, which is function of the effective electroelastic moduli $\mathbf{G}^{*}$ of matrix and the grain shape.

\section{ORIENTATION DISTRIBUTION FUNCTION}

It is clear that the estimation of the effective electroelastic moduli of piezoelectric polycrystals involves orientational volume averages, irrespective of the micromechanics scheme used. The orientation distribution of grains in the polycrystal can be described by Orientation Distribution Function (ODF) $W(\xi, \varphi, \phi){ }^{8}$, which is the probability density function for a grain at orientation $(\theta, \varphi, \phi)$. The orientational volume average of a single crystal tensorial property $\mathbf{H}$ weighted by an ODF is:

$$
<\mathbf{H}>=\int_{0}^{2 \pi} \int_{0}^{2 \pi} \int_{-1}^{1} \mathbf{H}(\xi, \varphi, \phi) W(\xi, \varphi, \phi) d \xi d \varphi d \phi
$$

where $\mathbf{H}(\xi, \varphi, \phi)$ is the single crystal value of $\mathbf{H}$ expressed in the global coordinate system. To evaluate $<\mathbf{H}>$, we expand both $\mathrm{W}(\xi, \varphi, \phi)$ and $\mathbf{H}(\xi, \varphi, \phi)$ into a series of generalized associated Legendre functions:

$$
\begin{aligned}
& W(\xi, \varphi, \phi)=\sum_{l=0}^{l=\infty} \sum_{m=-l}^{l} \sum_{n=-l}^{l} W_{l m n} Z_{l m n}(\xi) e^{-i m \varphi} e^{-i n \phi}, \\
& \mathbf{H}(\xi, \varphi, \phi)=\sum_{l=0}^{l=\infty} \sum_{m=-l}^{m=l} \sum_{n=-l}^{n=l} \mathbf{H}_{l m n} Z_{l m n}(\xi) e^{-i m \varphi} e^{-i n \phi},
\end{aligned}
$$

where $Z_{l m n}(\xi)$ are generalized associated Legendre functions. Due to orthogonality, the expansion coefficients can be expressed as:

$$
\begin{aligned}
& W_{l m n}=\frac{1}{4 \pi^{2}} \int_{0}^{2 \pi 2 \pi} \int_{0}^{1} \int_{-1}^{1} W(\xi, \varphi, \phi) Z_{l m n}(\xi) e^{i m \varphi} e^{i n \phi} d \xi d \varphi d \phi, \\
& \mathbf{H}_{l m n}=\frac{1}{4 \pi^{2}} \int_{0}^{2 \pi} \int_{0}^{2 \pi} \int_{-1}^{1} \mathbf{H}(\xi, \varphi, \phi) Z_{l m n}(\xi) e^{i m \varphi} e^{i n \phi} d \xi d \varphi d \phi .
\end{aligned}
$$

The texture coefficients $W_{l m n}$ represent the orientation distribution of grains in the polycrystalline aggregate. Even-rank texture coefficients can be determined from X-ray diffraction intensity as discussed in detail by Roe ${ }^{8}$. Such techniques are not able to provide information regarding odd-rank texture coefficients, due to the inherent centrosymmetry in X-ray diffraction. We suggest that this work may provide a potential method to determine the odd-rank texture coefficients inversely, from the measurement of odd-rank tensorial properties, such as the piezoelectric constants. Using eqs. (14) and (15), and taking into account the orthogonal property of $Z_{l m n}(\xi)$, equation (11) reduces to:

$$
<\mathbf{H}>=4 \pi^{2} \sum_{l=0}^{R} \sum_{m=-l}^{l} \sum_{n=-l}^{l} \mathbf{H}_{l m n} W_{l m n},
$$

where $\mathrm{R}$ is the rank of tensorial property $\mathbf{H}$. Only the first $\mathrm{R}$ terms in the series expansion need to be considered in averaging a tensorial property of rank $\mathrm{R}^{16}$. 


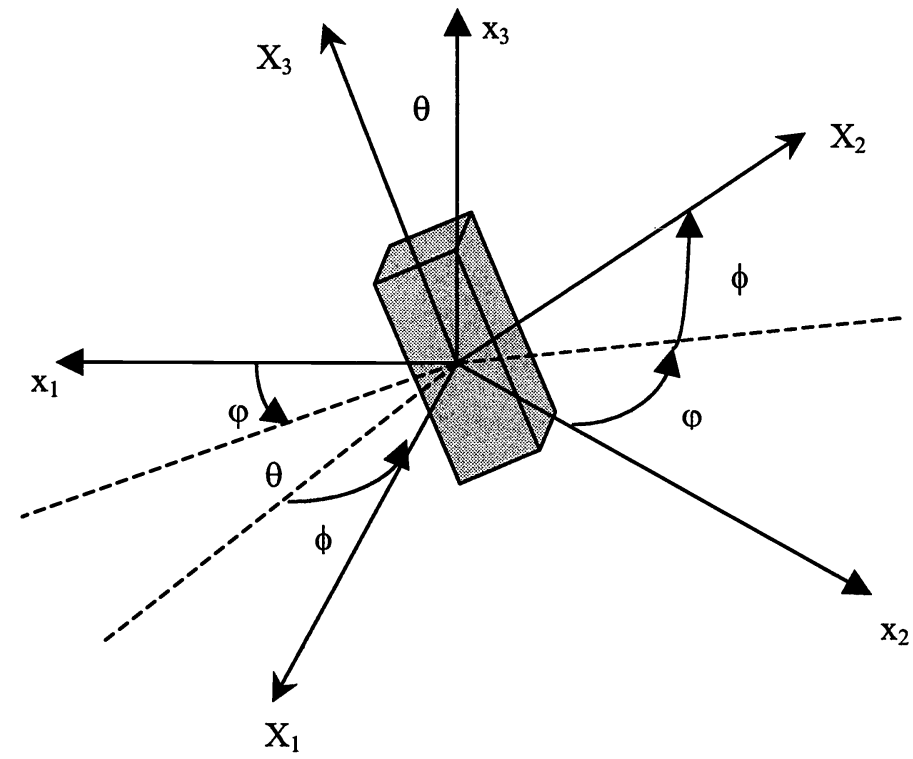

Figure 1. Euler angles $(\theta, \varphi, \phi)$ for a grain in a global coordinate system.

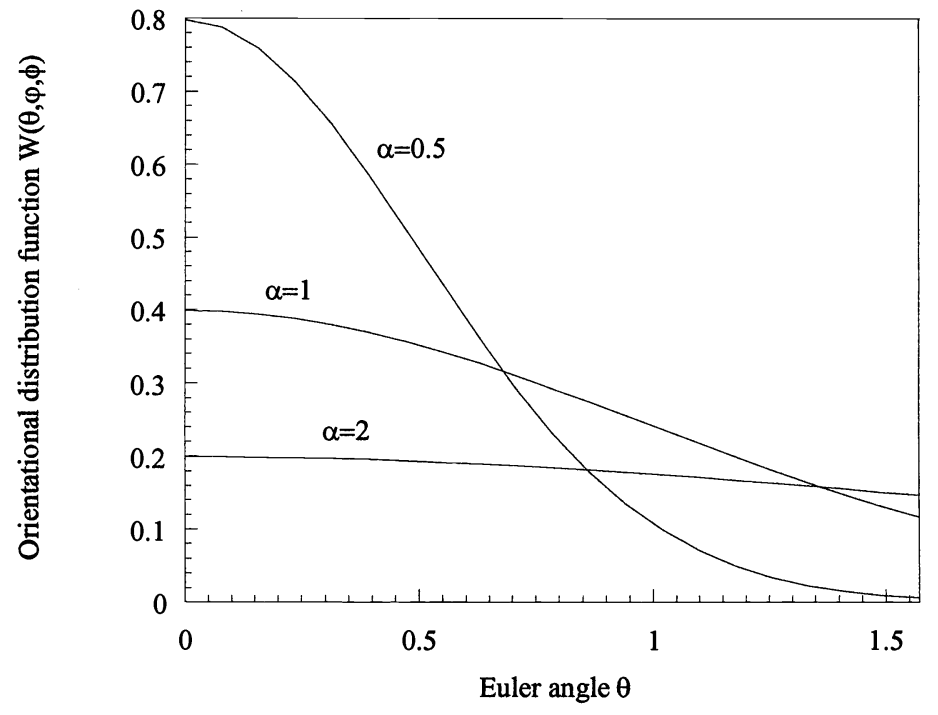

Figure 2. Gaussian distribution function with different parameter $\alpha$. 
In a compromise between simplicity and usefulleness, we approximate the ODF using a Gaussian distribution function. Because piezoelectric polycrystals are often transversely isotropic, we assume the ODF only depends on the Euler angle $\theta$, i.e.,

$$
W(\theta, \varphi, \phi)=\frac{1}{\alpha \sqrt{2 \pi}} \operatorname{Exp}\left(-\frac{\theta^{2}}{2 \alpha^{2}}\right)
$$

where the parameter $\alpha$ in the Gaussian distribution function depends on the details of the crystallographic texture. The shape of the Gaussian distribution function with various values of $\alpha$ is shown in Fig. 2. Two extreme cases follow : a polycrystal with perfectly aligned grains, such as a piezoelectric thin film, is realized by letting $\alpha \rightarrow 0$; and, a polycrystal with randomly oriented grains, such as an isotropic (unpoled) ceramic, is realized by letting $\alpha \rightarrow \propto$. Other textures can be realized by varying $\alpha$ in between these limits. In practice $\alpha$ is closely related to the processing details for the piezoelectric polycrystal, such as the

poling field intensity. For example, before poling the ceramic is isotropic, and $\alpha$ approaches infinity. When an electric field is applied during poling, some grains, or more precisely, subgrain domains, will reorient to minimize the free energy of the system. This can be represented by $\alpha$ takeing on a finite value, depending on the magnitude of the poling field. For a thin film with fiber texture, at the saturation field all of the grains are perfectly aligned with a polarization along the poling field direction, and $\alpha$ approaches zero. Via an appropriate measurement program, the relationship between $\alpha$ and the processing conditions can potentially be identified.

\section{POLYCRYSTALS WITH FIBER TEXTURE}

As a specific example, we consider piezoelectric polycrystals with a fiber texture where all grains in the polycrystal have a common symmetry axis aligned along the $\mathrm{x}_{3}$ direction (normal to the plane for a planar film). For this class of materials, the individual grains respond in exactly the same way to the applied loading under certain conditions, regardless of their orientations, so that the electroelastic fields in the polycrystal are uniform, and the effective electroelastic moduli can be found exactly. To pursue the conditions for such uniform fields, consider a single crystal, possessing a six-, four-, three-, or two-fold symmetry axis parallel to the $\mathrm{x}_{3}$ axis. We now consider simple electroelastic loadings of the polycrystal, where only one of the nine $\varepsilon_{\mathrm{p}}$ or $\mathrm{E}_{\mathrm{i}}$ is nonzero, and determine under what conditions the corresponding strain and electric displacement states in individual grains are invariant with respect to a rotation $\beta$ about the $x_{3}$ direction of the grain. Since all grains have the same symmetry axis aligned along the $x_{3}$ direction, this invariance means that all grains respond in an identical way to the external loading. In such a situation, the grains do not disturb each other, so no elastic and electric field fluctuations develop. The resulting electroelastic fields are invariant under the rotation $\beta$ around the $x_{3}$ direction if, and only if, the electroelastic moduli involved in a particular loading do not change under such a rotation, so that they are identical before and after the tensor transformation. The exact solutions for the effective electroelastic moduli under a particular rotational symmetry are summarized in Table 1. Complete details of the analysis are given elsewhere ${ }^{2}$.

\section{NUMERICAL RESULTS AND DISCUSSION}

To demonstrate the application of the theory we consider $\mathrm{BaTiO}_{3}$ polycrystals, where the single-crystal material constants ${ }^{17}$ are listed in Table 2 . We first apply the theory to a $\mathrm{BaTiO}_{3}$ polycrystal with texture described by the Gaussian distribution, with grains that are assumed to be spherical. Fig. 3 shows the effective piezoelectric constants of the $\mathrm{BaTiO}_{3}$ polycrystal as a function of $\alpha$. When $\alpha$ approaches zero, the grains in polycrystal become aligned. In this case the elastic constants $\mathrm{C}_{13}, \mathrm{C}_{33}$, and $\mathrm{C}_{44}$, and all of the piezoelectric and dielectric constants of the polycrystalline aggregate are exact and reduce to the corresponding single crystal values. As shown in Fig. 3, the Voigt-Reuss averages and the self-consistent predictions agree with each other and recover the single crystal values for these moduli. When $\alpha$ approaches infinity, the grains are randomly oriented and the polycrystal becomes isotropic and non-piezoelectric; in Fig. 3 It is seen that all piezoelectric constants become zero. Between these two extreme cases, the effective piezoelectric moduli show a strong dependency on texture, and there are peaks in piezoelectric constants $e_{31}$ and $e_{33}$ as $\alpha$ approaches $0.6-0.7$; the polycrystal constants are larger in magnitude than their single crystal values. This suggests that the piezoelectric properties of the polycrystal can be optimized by engineering the microstructure. Although not studied in detail here, we note that the same type of calculations can be applied at the domain level of a single crystal, suggesting again the optimization of certain material properties via microstructure tailoring. 
Table 1 Exact solutions for the thermoelectroelastic moduli for polycrystals with rotational symmetry around the $\mathrm{x}_{3}$ axis.

\begin{tabular}{lllll}
\hline Symmetry & Elastic & Piezoelectric & Dielectric & Thermal \\
\hline 6-fold & All & All & All & All \\
4-fold & $\mathrm{C}_{13}=\mathrm{C}_{23}, \mathrm{C}_{55}=\mathrm{C}_{44}, \mathrm{C}_{33}$ & $\mathrm{e}_{14}=-\mathrm{e}_{25}, \mathrm{e}_{15}=\mathrm{e}_{24}, \mathrm{e}_{31}=\mathrm{e}_{32}, \mathrm{e}_{33}$ & $\kappa_{11}=\kappa_{22}, \kappa_{33}$ & $\Delta_{1}=\Delta_{2}, \Delta_{3}$ \\
3-fold & $\mathrm{C}_{13}=\mathrm{C}_{23}, \mathrm{C}_{33}$ & $\mathrm{e}_{31}=\mathrm{e}_{32}, \mathrm{e}_{33}$ & $\kappa_{33}$ & $\Delta_{1}=\Delta_{2}, \Delta_{3}$ \\
2-fold & None & None & None & None \\
\hline
\end{tabular}

Table 2 Electroelastic moduli of tetragonal $\mathrm{BaTiO}_{3}$ single crystal

\begin{tabular}{llllll}
\hline $\mathrm{C}_{11}(\mathrm{GPa})$ & $\mathrm{C}_{12}(\mathrm{GPa})$ & $\mathrm{C}_{13}(\mathrm{GPa})$ & $\mathrm{C}_{33}(\mathrm{GPa})$ & $\mathrm{C}_{44}(\mathrm{GPa})$ & $\mathrm{C}_{66}(\mathrm{GPa})$ \\
\hline 275.1 & 178.9 & 151.55 & 164.8 & 54.3 & 113.1 \\
$\mathrm{e}_{31}\left(\mathrm{C} / \mathrm{m}^{2}\right)$ & $\mathrm{e}_{33}\left(\mathrm{C} / \mathrm{m}^{2}\right)$ & $\mathrm{e}_{15}\left(\mathrm{C} / \mathrm{m}^{2}\right)$ & $\kappa_{11} / \kappa^{0}$ & $\kappa_{33} / \kappa^{0}$ & \\
-2.69 & 3.65 & 21.3 & 1970 & 109 & \\
\hline
\end{tabular}

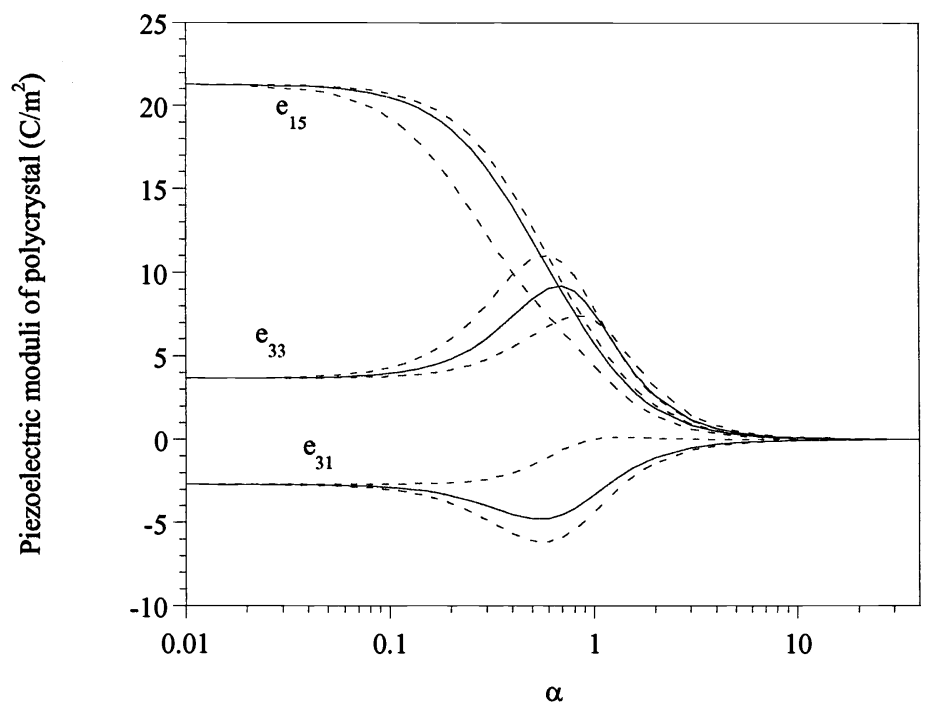

Figure 3. Effective piezoelectric moduli of a $\mathrm{BaTiO}_{3}$ piezoelectric polycrystal as a function of the Gaussian distribution function parameter $\alpha$. The solid line is the self-consistent approach, and the broken lines are Voigt-Reuss Averages. 


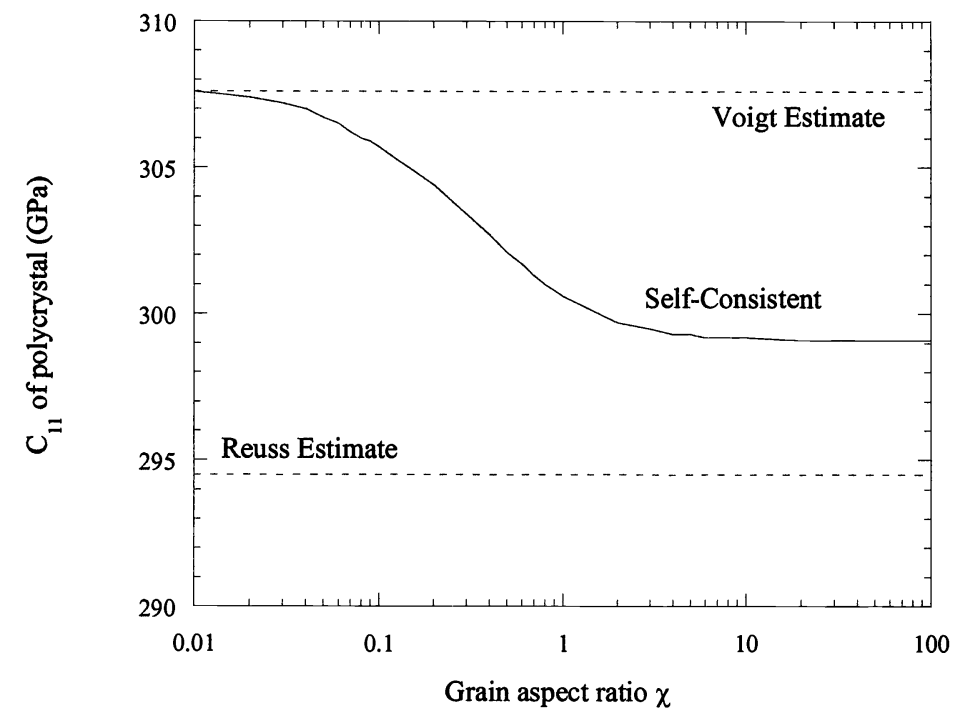

Figure 4. Effective elastic modulus $\mathrm{C}_{11}$ of a $\mathrm{BaTiO}_{3}$ piezoelectric polycrystal with perfect aligned grains as function of grain shape aspect ratio $\chi$.

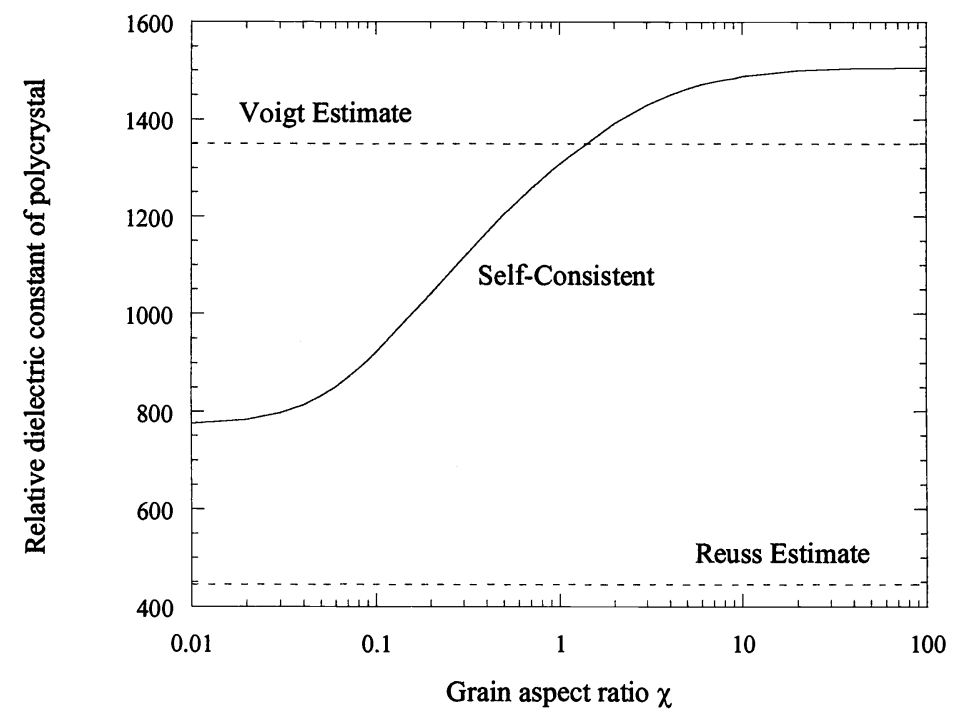

Figure 5. Effective relative dielectric constant $\mathrm{\kappa}$ of a $\mathrm{BaTiO}_{3}$ piezoelectric polycrystal with random aligned grains as function of grain shape aspect ratio $\chi$. 
For polycrystals with all $\mathrm{BaTiO}_{3}$ grains perfectly aligned in the $\mathrm{x}_{3}$ axis $(\alpha=0)$, for example, $\mathrm{BaTiO}_{3}$ thin films with columnar grains, the effective electroelastic moduli of the polycrystal are exact, and recover the single crystal values, except for $\mathrm{C}_{11}$ and $\mathrm{C}_{66}$. Our calculations confirm this result and show that the self-consistent approach recovers the exact solutions in this case. Fig. 4 shows the effective elastic constants $\mathrm{C}_{11}$ of such a $\mathrm{BaTiO}_{3}$ polycrystal as function of the grain shape, where the grains are assumed to be spheroidal and the shape is described by the aspect ratio, $\chi=a_{3} / a_{1}$. The polycrystal $\mathrm{C}_{11}$ are not very sensitive to grain shape; they change less than ten percent as the grain shape changes from penny-shape to needle-shape. It is highest for penny-shape grains, and decreases monotonically with the increase of the aspect ratio, reaching a minimum when the grains become needle-shaped. It is noted that Voigt-Reuss averages are unable to model the effect of grain shapes.

Polycrystals with randomly oriented $\mathrm{BaTiO}_{3}$ grains $(\alpha \rightarrow \infty)$ are isotropic and non-piezoelectric ${ }^{12,18}$. In this case, the independent overall electroelastic moduli are the bulk modulus $\mathrm{K}$, the shear modulus $\mathrm{G}$, and the dielectric constant $\kappa$. Although not shown, the effective elastic constants show a weak dependency on the grain shape. The dielectric constant, however, strongly depends on the grain shape, as shown in Fig. 5. It is a minimum for penny-shaped grains, increases monotonically with grain aspect ratio, and reaches a maximum for needle-shaped grains. The stronger dependence of dielectric constants on grain shape is believed to be caused by the stronger grain anisotropy in dielectric constants, as shown in Table 2.

\section{CONCLUSIONS}

The self-consistent approach along with the Voigt-Reuss estimates have been generalized to model the electroelastic moduli of piezoelectric polycrystals with texture. An orientational averaging scheme has been implemented using an orientation distribution function, which can describe general grain, or domain, orientations. For simplicity, we have approximated the ODF using a Gaussian distribution function, enabling simulation of a wide range of textures in polycrystals. Micromechanics estimates for $\mathrm{BaTiO}_{3}$ polycrystals have been presented, and shown to agree with exact results in special cases. The electroelastic moduli of piezoelectric polycrystalline aggregates show a strong dependence on crystallographic texture. Piezoelectric constants higher than single crystal values can be achieved at certain texture, and the dielectric constants are more sensitive to grain shape than the elastic constants due to the stronger electrical anisotropy.

For polycrystals with fiber texture, we have determined the conditions that permit exact solutions for some electroelastic moduli. While our study has focused on linear behavior, the basic framework can be used to describe the nonlinear constitutive response of a ferroelectric ceramic by incorporating the ODF coefficients ( $\alpha$ for the Gaussian ODF) as internal state variables in a constitutive model $^{4-6}$; this would enable, for example, the simulation of texture evolution during poling.

\section{REFERENCES}

1. Li, J. Y, "The Effective Electroelastic Moduli of Textured Piezoelectric Polycrystalline Aggregates," J. Mech. Phys. Solids 48, pp. 529-552, 2000.

2. Li, J. Y., Dunn, M. L., and Ledbetter, H., "Thermoelectroelastic Moduli of Textured Piezoelectric Polycrystals: Exact Solutions and Bounds for Film Textures," J. Appl. Phys. 86, pp. 4626-4634, 1999.

3. Nan, C. W. and Clarke, D. R., "Piezoelectric Moduli of Piezoelectric Ceramics," Journal of American Ceramic Society 79, pp. 2563-2566, 1996.

4. Huber, J. E., Fleck, N. A., Landis, C. M., McMeeking R. M., "A Constitutive Model for Ferroelectric Polycrystals,” J. Mech. Phys. Solids 47, pp. 1663-1697, 1999.

5. Li, J., Weng, G. J., "A Theory of Domain Switch for the Nonlinear Behaviour of Ferroelectrics," Proceedings of the Royal Society of London A455, pp. 3493-3511, 1999.

6. Rodel, J, Kreher, W. S., "Effective Properties of Polycrystalline Piezoelectric Ceramics," Journal de Physique IV 9, pp. 239-247, 1999.

7. Nye, J.F., Physical Properties of Crystals, Oxford University Press, Oxford, UK, 1957.

8. Roe, R. J., "Description of Crystalline Orientation in Polycrystalline Materials, III. General Solution to Pole Figure Inversion," J. Appl. Phys. 36, pp. 2024-2031, 1965.

9. Voigt, W., "Ueber die Beziehung zwischen den beiden Elastizita tskonstanten isotroper Korper," Annalen der Physik 38, pp. 573-587, 1889.

10. Reuss, A., "Berechnung der Fliessgrenze von Mischkristallen auf Grund der Plastizita tsbedingung ur Einkristalle," Zeitschrift fur angewandte Mathematik und Mechanik 9, pp. 49-58, 1929.

11. Willis, J. R., "Bounds and Self-consistent Estimates for the Overall Properties of Anisotropic Composites," J. Mech. Phys. Solids 25, pp. 185-202, 1977. 
12. Dunn, M. L., "Effects of Grain Shape Anisotropy, Porosity, and Microcracks on the Elastic and Dielectric Constants of Polycrystalline Piezoelectric Ceramics," J. Appl. Phys. 78, pp. 1533-1542.

13. Eshelby, J. D., "The Determination of the Elastic Field of an Ellipsoidal Inclusion, and Related Problems," Proceedings of the Royal Society of London A241, pp. 376-396, 1957.

14. Dunn, M. L. and Taya, M., "An Analysis of Composite Materials Containing Ellipsoidal Piezoelectric Inhomogeneities," Proceedings of the Royal Society of London A443, pp. 265-287, 1993.

15. Dunn, M. L. and Wienecke, H. A., "Inclusions and inhomogeneities in transversely isotropic piezoelectric solids," Int. J. Solids Struct. 34, pp. 3571-3582, 1997.

16. Ferrari, M. and Johnson, G. C., "The Equilibrium Properties of a $6 \mathrm{~mm}$ Polycrystal Exhibiting Transverse Isotropy," $J$. Appl. Phys. 63, pp. 4460-4468, 1988.

17. Berlincourt, D. and Jaffe, H., "Elastic and Piezoelectric Coefficients of Single-Crystal Barium Titanate," Phys. Rev. B, 111, pp. 143-148, 1958.

18. Olson, T. and Avellaneda, M., "Effective Dielectric and Elastic Constants of Piezoelectric Polycrystals," J. Appl. Phys. 71, pp. 4455-4464 1992. 